\title{
Article \\ On the Optimal Design of Steel Shells with Technological Constraints
}

\author{
Dragana Turnić * , Tomislav Igić, Srđan Živković, Aleksandra Igić and Marija Spasojević Šurdilović
}

Faculty of Civil Engineering and Architecture, University of Nis, 18000 Niš, Serbia; tomislav.igic@gaf.ni.ac.rs (T.I.); srdjan.zivkovic@gaf.ni.ac.rs (S.Ž.); igic.aleksandra91@gmail.com (A.I.); spasojevic.n.marija@gmail.com (M.S.Š.)

* Correspondence: dragana.turnic@gaf.ni.ac.rs; Tel.: +381-642282598

\begin{abstract}
This paper is concerned with determining the optimum conditions of steel cylindrical shells with technological limitations and one behavioral constraint, related to a specific constitutive law for limiting load-carrying capacity. The optimum structural design in the plastic range of circular cylindrical full shells composed of rings of variable thickness is given. A numerical procedure for determining the optimal dimensions of shell rings is given. A shell collapse mechanism is assumed in the kinematic part, which satisfies requirements. Within the optimum conditions, the power of the dissipation energy of rings assuming the hexagon Hodge condition of plasticity are derived. A series of numerical solutions and results of optimal structural designs for a shell that is simply supported at the ends are presented. In one example of optimally calculated shells, the length $X_{1}$ of one ring was varied.
\end{abstract}

Keywords: steel shell; optimum design; optimum conditions; dissipation energy; plasticity

check for updates

Citation: Turnić, D.; Igić, T.; Živković, S.; Igić, A.; Spasojević Šurdilović, M. On the Optimal Design of Steel Shells with Technological Constraints. Appl. Sci. 2022, 12, 2282. https://doi.org/ 10.3390/app12052282

Academic Editors: Jong Wan Hu and Junwon Seo

Received: 13 January 2022

Accepted: 16 February 2022

Published: 22 February 2022

Publisher's Note: MDPI stays neutral with regard to jurisdictional claims in published maps and institutional affiliations.

Copyright: (c) 2022 by the authors. Licensee MDPI, Basel, Switzerland. This article is an open access article distributed under the terms and conditions of the Creative Commons Attribution (CC BY) license (https:// creativecommons.org/licenses/by/ $4.0 /)$.

\section{Introduction}

We consider the structural optimal design of a circular cylindrical shell composed of rings. The objective function is cost $C$ of the shell. Satisfying the condition of the minimal cost $C$, we obtained the minimal volume of the shell. First, we used the general structure optimization theory in the plastic region, first developed by Drucker, Shield, Prager, Save, and others. In this procedure, we determined the absolute minimum cost of an optimum structural design for real circular cylindrical shell structures. The problems highlighted here include limited analysis, optimum conditions of shells for yield condition, and optimum plastic dimensioning of the steel shells with rings of different thicknesses. When we consider the shell in the plastic area, in comparison to the elastic, there is a significant plastic reserve in the material and we are able to determine the value of the ultimate load when a fracture occurs. By optimizing for the same load, we obtained the minimum material, i.e., minimum shell volume.

Deriving the optimum conditions and the optimal dimensioning of the shells, we were able to minimize the power of dissipation energy under the given limit load that leads to plastic collapse.

For technological reasons, we treated the shell with rings because this makes it easier to perform in practice. We determined the optimal conditions with technological constraints. Thus, the thickness $e_{i}$ of the ring must be within the limits: minimum $e_{i \text {,inf }} \leq e_{i}$, and maximum $e_{i \text {,sup }} \geq e_{i}$. In this way, we ensure that, in cross-sections, the shell thickness is large enough to satisfy the influence of shear forces. In certain cases, technological limitations can be introduced due to functionality reasons.

Often, designs that are difficult to execute (thickness may turn out to be exceedingly small), or that violate basic assumptions of the theory of shell optimization (far too great a thickness in comparison to diameter), are avoided.

In the optimum plastic design of structures, the theory is much more advanced than the experiments. In particular, this discrepancy refers to the optimal dimensioning of the 
shells, where new publications have been published [1,2]. Among others, the experiments of Kouam [3] in Guerlement's laboratory demonstrated the application of limit analysis theory for the optimization of cylindrical shells.

Engineers have a specific role in that they must satisfy all requirements regarding determining and dimensioning in industry and practice [4,5]. Structural optimum design requires an individualized approach, as no general method for solving these problems has been established. When optimizing a structure i.e., of its material, the principle of minimum potential deformation energy in elastic and minimum power of dissipation energy in plastic region is applied. In more complex cases, both ranges and two kinds of energies are minimized [6,7]. The commercial engineering optimization software packages based on the finite element method are currently used by researchers to solve standard and non-standard engineering problems. Recently, a large number of papers related to shell problems, as well as optimization in various fields, have emerged from both theoretical and numerical and technological and practical aspects [8].

Optimization is especially important when there is a need for a large series of the same elements or structures. Additionally, in certain cases, the requirements are that the weight of the construction must be as small as possible, i.e., optimal, for example, in the case of aircrafts. An increasing number of papers concerning this have appeared in the last decade. A small number of papers, due to the complexity of this problem, do not have analytical solutions, but most often apply the finite element method $[9,10]$. New directions for research have evolved in this field. In the paper [11], a purely numerical approach based on optimized numerical integration for probabilistic modeling of a fatigue crack progression is used. More complex shell structures are solved due to different types of loads related to the problem of stability-buckling and dynamics [12,13]. In [14], a non-linear formulation is derived for the assessment of a load of cylindrical shells subjected to pressure, which accounts for the onset of plasticity, including the effect of geometrical imperfections. Shell structures are very important in civil engineering, mechanical engineering, aerospace, and other disciplines. In paper [15], in the frame of the structural analysis of shell structures, the elastic region was extended to the plastic region and the formulation carried out in this paper includes large strain plastic deformation. The shells are represented in the petrochemical industry as shell-and-tube heat exchangers. The study in [16] focuses on the calculations for obtaining the fields of equivalent stresses and plastic deformations.

An optimal design of shells in the elastic region of various constraints has been implemented in [17]. The weight efficiency for various types of shells reinforcements was studied.

For practical, very complex problems concerning the interaction of two shells, as is the case in [18], an optimization method is presented, which combines a finite element method analysis and an optimization algorithm where load path optimization is performed to minimize rod force.

The method of genetic algorithms has been successfully applied to the problem of optimal dimensioning of rotationally symmetric shells [19]. Combining mathematical programming, the finite element method, and the limit analysis of shells, the limit load was calculated and the genetic algorithm led to research concerning the optimal shell using a search heuristic method.

To optimize steel shells in the plastic area considered in this paper, it is necessary to determine the limit analysis of the shell.

\section{Basic Equations for the Limit Analysis of Shells}

We observe a simple case of a circular cylindrical shell subjected to pressure $p(x)$ in the outward radial direction. A homogeneous and orthotropic material with rigid, perfectly plastic behavior is considered. The cylindrical coordinate system $(r, \theta, x)$ is chosen with the axis $x$, along with the symmetry. All functions depend on $x$ only. The only displacement of the median surface of the shell is in the radial direction. For the assumption that the 
elements normal to the median surface are straight and normal after deformation, see Figure 1.

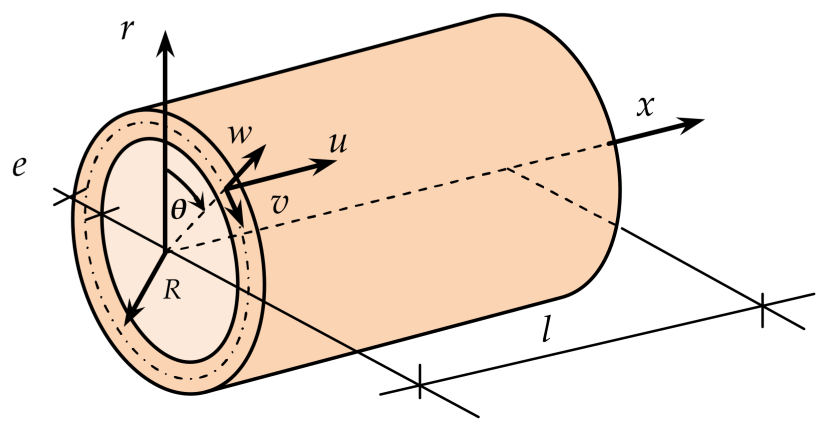

Figure 1. Circular cylindrical shell.

The power of dissipation energy $\mathcal{D}$ across a shell element is given by

$$
\mathcal{D}=\int_{-e / 2}^{+e / 2}\left(\sigma_{x} \dot{\varepsilon}_{x}+\sigma_{0} \dot{\varepsilon}_{\theta}\right) d z
$$

$\dot{\varepsilon}_{\theta}$ and $\dot{\varepsilon}_{x}$ are the strain rates of unit circular and axial lengths. It follows as

$$
\mathcal{D}=\frac{d^{2} \dot{w}}{d x^{2}} M_{x}+\frac{\dot{w}}{R} N_{\theta}
$$

where the stress resultants $M_{x}$ and $N_{\theta}$ are introduced (See Figure 2). Introducing the dimensionless values $m_{x}$ and $n_{\theta}$, i.e.

$$
m_{x}=\frac{M_{x}}{M_{p x}}, n_{\theta}=\frac{N_{\theta}}{N_{p \theta}}
$$

where

$$
M_{p x}=\frac{\sigma_{0} e^{2}}{4}, N_{p \theta}=\sigma_{0} e .
$$

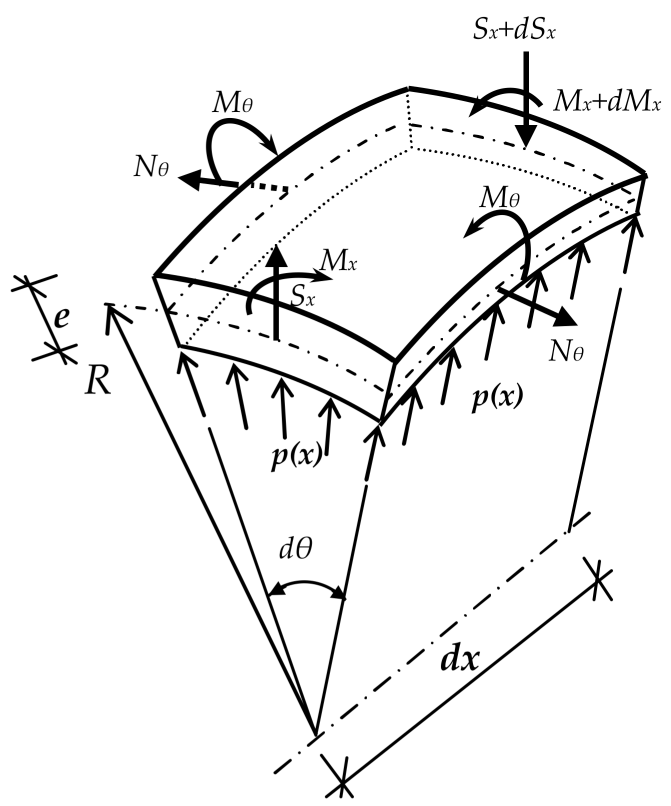

Figure 2. Shell differential element. 
The dissipation $\mathcal{D}$ from (1) is given

$$
\mathcal{D}=\sigma_{0} e\left(\dot{k}_{x} m_{x}+\dot{\lambda}_{\theta} n_{\theta}\right) .
$$

The strain rates $\dot{k}_{x}$ and $\dot{\lambda}$ corresponding to the generalized stresses $m_{x}$ and $n_{\theta}$ respectively are the generalized strain rates

$$
\dot{k}_{x}=\frac{e}{4} \frac{d^{2} \dot{w}}{d x^{2}}, \dot{\lambda}_{\theta}=\frac{\dot{w}}{R}
$$

where $\dot{w}$ is the displacement rate of the median surface of the shell.

The equilibrium of the shell differential element leads to the differential equation

$$
\frac{d^{2} M_{x}}{d x^{2}}+\frac{N_{\theta}}{R}-p(x)=0 .
$$

By denoting $M_{x}$ and $N_{\theta}$ from (3), Equation (7) reads

$$
\frac{d^{2} m_{x}}{d x^{2}}+2 \omega^{2}\left(n_{\theta}-\bar{p}\right)=0
$$

where

$$
\omega^{2}=\frac{2}{e R}, \bar{p}=p \frac{R}{\sigma_{0} e} .
$$

Hodge's yield condition. The yield condition shows the points of the structure where stresses $M_{x}$ and $N_{\theta}$ are in a state of plasticity. Unlike the previous ones, the stresses $M_{\theta}$ and $T_{x}$ do not appear in the dissipation and represent a reaction correlating to the work by Prager [7]. Starting from Tresca's material, we obtained a nonlinear interaction curve. On the suggestion of Drucker, the linear approximation was made [20]. Using dimensionless variables $n_{\theta} m_{x}$, the result was Hodge's hexagon (see Figure 3).

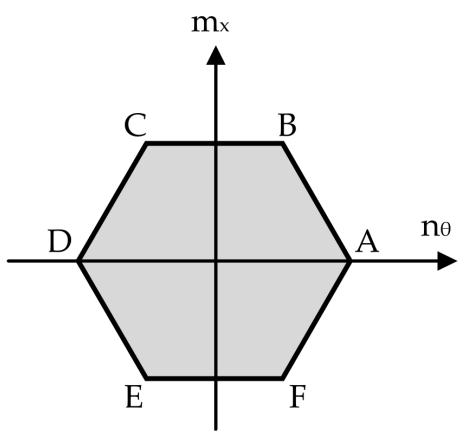

Figure 3. Hodge's plastic yield condition.

For a shell element in a limited state, the stresses are represented by a point on Hodge's hexagon. The sides of the hexagon provide the relationship variables $m$ and $n$ given in Hodge's Table 11-1 in [21] (p. 277). For each side of the hexagon, we find $\dot{w}$ with (6), $\dot{w}$ is a continuous function.

\section{Conditions of Minimum Cost Plastic Design of Full Steel Cylindrical Shells}

\subsection{Hypothesis}

Let there be the cylindrical shell (Figure 4) whose middle surface area and boundary conditions are known. The material which constitutes a rigid plastic shell has the yield stress $\sigma_{0}$. Shell layout is divided into $n$ rings bound by the known abscissas. The technological constraints are as follows: $e_{i, \text { inf }} \leq e_{i} \leq e_{i, \text { sup }}$. The problem can be expressed in the following way: 


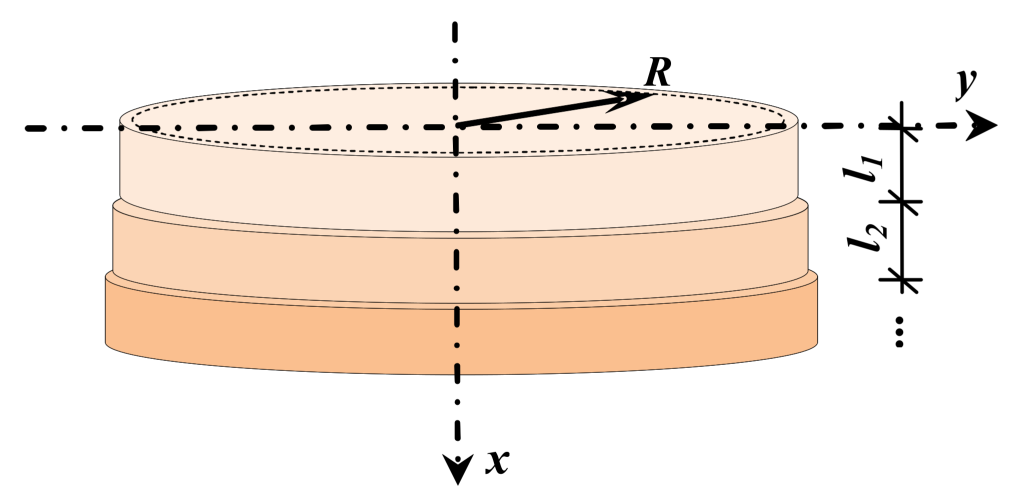

Figure 4. The cylindrical shell formed by rings.

The total cost $C$ of the shell is given as

$$
C=\sum_{i=1}^{n} A_{i} c_{i}
$$

where $A_{i}=2 \pi R l_{i}, i=1, \ldots, n$ and $c_{i}$ is specific cost, i.e., cost per unit of middle surface area of the ring number $i$. We assume that the specific cost is a function of the thickness of the ring, $e_{i}$. The problem of the optimal plastic design of the shell is set as follows: find the set of values $e_{i}(i=1, \ldots, n)$ such that the shell is exactly on the verge of collapse under load $p(x)$, to meet technological constraints and the total cost $C$ of the shell (the objective function) be minimal.

We assume that the specific $\operatorname{cost} c_{i}$ is the function of thickness $e_{i}$.

Suppose without loss of generality, an external, uniformly distributed load $p(x)$. As is usual for thin shells, volume forces are ignored. The stresses in the shell due to volume forces are many times lower than the stresses caused by the limit load where the yield stress of steel is $\sigma_{0}=235 \mathrm{MPa}$.

\subsection{Sufficient Optimality Conditions for the Full Shell Case}

The structural design is expressed through the shell thicknesses of the rings, $e_{i}$. The shell under load $p(x)$ is exactly on the verge of collapse. The collapse mechanism describes a displacement velocity field. Using the combined theorem of the limit analysis, we can write

$$
\int_{A} p(x) \dot{w}(x) \mathrm{d} A=\sum_{i=1}^{n} D_{i}
$$

Let a neighboring design $e_{i}^{*}=e_{i}+\delta e_{i}$ meet technological constraints so that it can at least sustain a given load. The upper bound theorem of the limit analysis implies

$$
\int_{A} p(x) \dot{w}(x) \mathrm{d} A \leq \sum_{i=1}^{n} \mathcal{D}_{i}^{*}=\sum_{i=1}^{n} \mathcal{D}_{i}+\sum_{i=1}^{n} \delta e_{i} \frac{\partial \mathcal{D}_{i}}{\partial e_{i}} .
$$

The field of displacements rates $\dot{w}_{x}^{(i)}$ is added to the dimensioning $e_{i}$, but is only allowed for dimensioning $e_{i}^{*}$ and therefore an inequality sign is established. The specific power of dissipation energy $\mathcal{D}_{i}[\dot{w}(x)]$ is assumed to be a continuously differentiable function at $e_{i}$. Thus, we have

$$
\sum_{i=1}^{n} \delta e_{i} \frac{\partial \mathcal{D}_{i}}{\partial e_{i}} \geq 0
$$


Suppose that the collapse mechanism, $\dot{w}(x)$, is such that

$$
\frac{\partial \mathcal{D}_{i}}{\partial e_{i}}=\left(\frac{d c}{d e}\right)_{\begin{array}{l}
e \\
e
\end{array}}=e_{i} A_{i}+t_{i}, i=1, \ldots, n,
$$

where $t_{i}, i=1, \ldots, n$, are constants and $A_{i}$ is the surface of the midline of the ring with thickness $e_{i}$. From (13) with (14) we have

$$
\sum_{i=1}^{n} \delta e_{i}\left(\frac{d c}{d e}\right)_{\begin{array}{l}
e \\
c
\end{array}=e_{i}} A_{i}+\sum_{i=1}^{n} \delta e_{i} t_{i} \geq 0, i=1, \ldots, n,
$$

Analyzing the previous relation, we obtain

$$
\begin{gathered}
\frac{\partial \mathcal{D}_{i}}{\partial e_{i}} \leq\left(\frac{d c}{d e}\right)_{\begin{array}{c}
e=e_{i} \\
c=c_{i}
\end{array}} A_{i}, \text { for } e_{i}=e_{i, \text { inf },} \\
\frac{\partial D_{i}}{\partial e_{i}} \geq\left(\frac{d c}{d e}\right)_{\substack{e=e_{i} \\
A_{i}}} \text {, for } e_{i}=e_{i, \text { sup }}, \\
\frac{\partial D_{i}}{\partial e_{i}}=\left(\frac{d c}{d e}\right)_{e=e_{i}} A_{i} \text {, for } e_{i, \text { inf }}<e_{i}<e_{i, \text { sup } .}
\end{gathered}
$$

For the Inequalities (16) and (17) were $\delta e_{i} t_{i} \leq 0$ and to obtain Equation (18) was $t_{i}=0$. For the previous analysis, the Inequality (15) can be written in a stronger form

$$
\sum_{i=1}^{n} \delta e_{i}\left(\frac{d c}{d e}\right)_{\begin{array}{l}
e \\
e
\end{array}}=e_{i} A_{i} \geq 0
$$

which indicates that the design $e_{i}^{*}$ includes a higher cost than design $e_{i}$.

The relationships (16), (17), and (18) represent relative minimum cost.

\subsection{Necessary Optimality Conditions for the Full Shell Case}

Let $e_{i}$ be the design that satisfies all the technological constraints, which is exactly on the verge of collapse due to external loads $p(x)$ and gives the minimum total $\operatorname{cost} C$ of the shell. Starting with (11) we make a dimensioning change $e_{i}$. The corresponding collapse mechanism obtains another strain displacement $\dot{w}$ as well as another limit load $p$. Thus, Equation (11) becomes

$$
\sum_{i=1}^{n}\left(\frac{\partial \mathcal{D}_{i}}{\partial e_{i}} \delta e_{i}+\frac{\partial \mathcal{D}_{i}}{\partial \dot{w}} \delta \dot{w}\right)=\int_{A}\left(\frac{\partial(p \dot{w})}{\partial \dot{w}} \delta \dot{w}+\frac{\partial(p \dot{w})}{\partial p} \delta p\right) \mathrm{d} A .
$$

Using the principle of virtual work,

$$
\sum_{i=1}^{n} \frac{\partial \mathcal{D}_{i}}{\partial \dot{w}} \delta \dot{w}=\int_{A} p(x) \delta \dot{w} \mathrm{~d} A,
$$

Equation (20) gives

$$
\sum_{i=1}^{n} \frac{\partial \mathcal{D}_{i}}{\partial e_{i}} \delta e=i \int_{A} \dot{w} \delta p \mathrm{~d} A
$$


From the assumption that $e_{i}$ represents an optimal design, we have

$$
\sum_{i=1}^{n} A_{i} \frac{\partial c_{i}}{\partial e_{i}} \delta e_{i} \geq 0 \text { if } \delta p=0
$$

Subtracting Equation (22) from the Inequality (23), we obtain

$$
\delta e_{i}\left(A_{i} \frac{\partial c_{i}}{\partial e_{i}}-\frac{\partial \mathcal{D}_{i}}{\partial e_{i}}\right) \geq 0 .
$$

Now, we have three possibilities:

1. If $e_{i}=e_{i, i n f}$, a variation $\delta e_{i}$ must be positive or equal to zero, and the inequality (24) is satisfied by (16)

2. If $e_{i}=e_{i, s u p}$, a variation $\delta e_{i}$ must be negative or equal to zero, and the Inequality (24) is satisfied by (17)

3. If $e_{i, \text { inf }}<e_{i}<e_{i, \text { sup }}$, a variation $\delta e_{i}$ must be positive or negative, and the Inequality (24) is satisfied by (18).

The conditions (16), (17), and (18) are necessary conditions of the optimal plastic design. They are identical to the sufficient conditions for the relative minimum cost of full shells composed of rings of different thicknesses and were first derived by Igić in [22].

\section{Optimal Plastic Design of Shell Composed of Three Rings}

In this section, we consider the optimal plastic design of a circular cylindrical full shell composed of three rings. The ring thicknesses are $e_{1}$ and $e_{2}$ were $e_{2} \geq e_{1}$. The shell is subjected to an axially symmetric radial uniform pressure distribution $p_{0}$ and no end load. The shell is simply supported. Assuming the perfect plasticity, the dimensioning will use Hodge's yield hexagon for cylindrical shells. The thicknesses $e_{1}$ and $e_{2}$ of the rings should be determined so that the shell is on the verge of collapse due to the given limit load $p_{0}$ and with total minimum volume. The mechanism of shell collapse is presented in Figure 5.

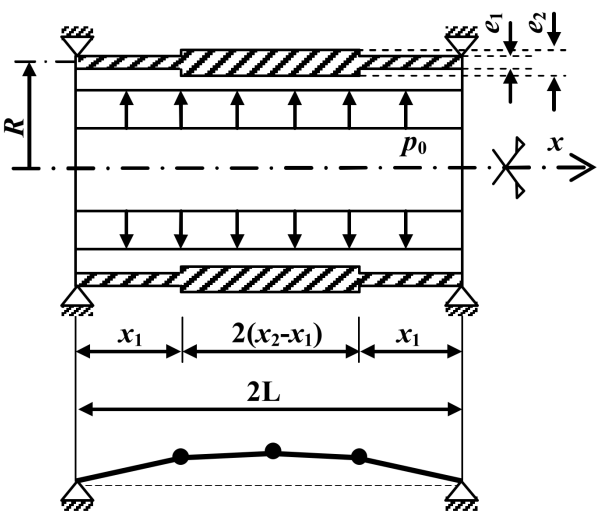

$$
\begin{aligned}
& 2 \mathrm{~L}=2 x_{2} \text {-total length of the shell, } \\
& x_{1} \text {-length of the ring near the support, } \\
& e_{1} \text {-thickness of the ring near the support, } \\
& e_{2} \text {-thickness of the central ring, } \\
& R \text {-radius of the cylindrical shell to the } \\
& \text { midline, } \\
& \sigma_{o}-\text { yield stress in tension, } \\
& p_{o}-\text { imposed limit loading. }
\end{aligned}
$$

Figure 5. The simply supported full shell composed of three rings and shell collapse mechanism.

Dimensioning. Based on the method for obtaining the required thicknesses $e_{1}$ and $e_{2}$ in the static part, we start with the equilibrium equations of the shell, assuming that the shell is on the verge of collapse. According to the plastic regime on Hodge's yield condition and expression for moments, we used fracture mechanics, support conditions, symmetry, and the fact that plastic hinges formed in the cross-section, between the rings, are on the side of the ring with smaller thickness $\mathrm{e}_{1}$.

Thus, six equations from which we determined the unknown ring thicknesses $e_{1}$ and $e_{2}$ and the unknown constants for the bending moments $M_{x}^{(1)}$ and $M_{x}^{(2)}$ on the part of the ring $e_{1}$ and $e_{2}$, respectively, were obtained. In addition to these six equations, we also have the equation of the relationship between the moments of plasticity of the rings. So, in these seven equations, we have seven unknowns. 


\subsection{Statically Admissible Solution}

Based on the static boundary conditions, the continuity of the section forces, and the conditions of symmetry, we obtained the following six equations

$$
\begin{aligned}
& x=0 ; M_{x}^{(1)}=0 ; \\
& x=x_{1} ; M_{x}^{(1)}=-\xi M p_{2} ; \\
& x=x_{1} ; \frac{d M_{x}^{(1)}}{d x}=\frac{d M_{x}^{(2)}}{d x} ; \\
& x=x_{1} ; M_{x}^{(2)}=-\xi M p_{2} ; \\
& x=x_{2}=L ; M_{x}^{(2)}=-M p_{2} ; \\
& x=x_{2}=L ; \frac{d M_{x}^{(2)}}{d x}=0 .
\end{aligned}
$$

Using Hodge's yield condition and Table 11-3 in [21] (p. 277) to our case under consideration, we can obtain for bending moment $M_{x}^{(i)}$, normal force $N_{\theta}^{(i)}$, and displacement rate $\dot{w}_{x}^{(i)}$, as follows

$$
\begin{gathered}
M_{x}^{(i)}=\frac{\sigma_{0} e_{i}^{2}}{2}\left(D_{3}^{(i)} \sin \omega^{(i)} x+D_{4}^{(i)} \cos \omega^{(i)} x+\overline{p_{0}}-1\right), \\
N_{\theta}^{(i)}=\sigma_{0} e_{i}\left(D_{3}^{(i)} \sin \omega^{(i)} x+D_{4}^{(i)} \cos \omega^{(i)} x+\bar{p}_{0}\right), \\
\dot{w}_{x}^{(i)}=D_{1}^{(i)} \sin \omega^{(i)} x+D_{2}^{(i)} \cos \omega^{(i)} x,
\end{gathered}
$$

where $D_{1}^{(i)} D_{2}^{(i)}$ and are the constants and $\omega^{(i)}$ is a parameter from (9). Using the parameter $\xi$, the relationship moments of plasticity of rings $M_{p x}^{(i)}=\frac{\sigma_{0} e_{i}^{2}}{4},(i=1,2)$ is defined by

$$
\xi \cdot M_{p x}^{(2)}=M_{p x}^{(1)}, \text { i.e., } \xi \cdot e_{2}^{2}=e_{1}^{2} .
$$

The system Equations (25) with (26) and (29) reads

$$
\begin{aligned}
& \frac{\sigma_{0} e_{1}^{2}}{2}\left(D_{3}^{(1)} \sin \omega^{(1)} x+D_{4}^{(1)} \cos \omega^{(1)} x+\bar{p}^{(1)}-1\right)=0 ; \\
& \frac{\sigma_{0} e_{1}^{2}}{2}\left(D_{3}^{(1)} \sin \omega^{(1)} x_{1}+D_{4}^{(1)} \cos \omega^{(1)} x_{1}+\bar{p}^{(1)}-1\right)=-\xi^{\sigma_{0} e_{2}^{2}} \\
& \frac{\sigma_{0} e_{1}^{2}}{2} \omega^{(1)}\left(D_{3}^{(1)} \cos \omega^{(1)} x_{1}-D_{4}^{(1)} \sin \omega^{(1)} x_{1}\right)=\frac{\sigma_{0} e_{2}^{2}}{2} \omega^{(2)}\left(D_{3}^{(2)} \cos \omega^{(2)} x_{1}-D_{4}^{(2)} \sin \omega^{(2)} x_{1}\right) ; \\
& \frac{\sigma_{0} e_{2}^{2}}{2}\left(D_{3}^{(2)} \sin \omega^{(2)} x_{1}+D_{4}^{(2)} \cos \omega^{(2)} x_{1}+\bar{p}^{(2)}-1\right)=-\xi^{\frac{\sigma_{0} e_{2}^{2}}{4}} \\
& \frac{\sigma_{0} e_{2}^{2}}{2}\left(D_{3}^{(2)} \sin \omega^{(2)} x_{2}+D_{4}^{(2)} \cos \omega^{(2)} x_{2}+\bar{p}^{(2)}-1\right)=-\frac{\sigma_{0} e_{2}^{2}}{4} \\
& \frac{\sigma_{0} e_{2}^{2}}{2} \omega^{(2)}\left(D_{3}^{(2)} \cos \omega^{(2)} x_{2}-D_{4}^{(2)} \sin \omega^{(2)} x_{2}\right)=0,
\end{aligned}
$$

where

$$
\bar{p}^{(i)}=\frac{p_{0} R}{\sigma_{0} e_{i}}, \omega^{(i)}=\sqrt{\frac{2}{e_{i} R}} .
$$

We introduce the dimensionless values

$$
P_{s}=\frac{p_{0}}{\sigma_{0}}, E_{i}=\frac{e_{i}}{R}, X_{i}=\frac{x_{i}}{R}
$$


The system (30) and (29) contains seven unknown variables $e_{1}, e_{2}$, constants $D_{3}^{(1)}, D_{4}^{(1)}$, $D_{3}^{(2)}, D_{4}^{(2)}$ and the parameter $\xi$. After eliminating constants, three nonlinear equations with unknowns $e_{1}, e_{2}$, and $\xi$ were obtained

$$
\begin{gathered}
\left(\frac{1}{2}-\frac{P_{s}}{E_{2}}\right) \cdot\left\{\cos \left[\left(X_{2}-X_{1}\right) \sqrt{\frac{2}{E_{2}}}\right]-1\right\}+\frac{\xi}{2}-\frac{1}{2}=0 \\
{\left[1-\frac{P_{s}}{E_{1}}-\frac{\xi}{2}\left(\frac{E_{2}}{E_{1}}\right)^{2}\right] \cos \left(X_{1} \sqrt{\frac{2}{E_{1}}}\right)-\left(1-\frac{P_{s}}{E_{1}}\right)-\left(\frac{E_{2}}{E_{1}}\right)^{2} \sqrt{\frac{E_{1}}{E_{2}}}\left(0.5-\frac{P_{s}}{E_{2}}\right) \cdot \sin \left(X_{1} \sqrt{\frac{2}{E_{1}}}\right) \sin \left[\left(X_{2}-X_{1}\right) \sqrt{\frac{2}{E_{2}}}\right]=0} \\
\xi \cdot E_{2}^{2}=E_{1}^{2}
\end{gathered}
$$

By introducing $E_{1}=E_{2}$, then $X_{2}=X_{1}$ and $\xi=1$ into (34), this Equation (34) becomes the equation of the shell of constant thickness $E_{1}$, i.e.,

$$
\left(\frac{1}{2}-\frac{P_{s}}{E_{1}}\right) \cos \left(X_{1} \sqrt{\frac{2}{E_{1}}}\right)+\frac{P_{s}}{E_{1}}-1=0 .
$$

To solve this system of nonlinear equations, a numerical procedure was implemented. Knowing that the ratio of moments of plasticity is as in (29) and $E_{1} \leq E_{2}$, the variable $\xi$ must be in the interval $0 \leq \xi \leq 1$. In a computer program, created according to that procedure, we reduce $\xi$ by a fixed step $h$. The main program contains two subroutines adopted for the numerical calculation of nonlinear equations. In the first subroutine, we determine $E_{2}$ and with this value, we obtain $E_{1}$ from (34). By entering the values $E_{1}$ and $E_{2}$, calculated in the first step, into the main program is it checked whether the condition (35) is met. If this condition is not met, we reduce the initial interval for $\xi$ by the initial step $h=0.1$ and $n=10$. Then, the procedure is repeated with a new step $h_{1}=\frac{h}{n}=\frac{1}{n^{2}}$, etc. We interrupt the procedure $h_{m}=\frac{1}{n^{m+1}}<\varepsilon$. In this way, the statically admissible solutions, $E_{1}$ and $E_{2}$, were obtained. In Sections 5 and 6, Example 1, a part of this procedure will be shown.

Knowing the values of $E_{1}, E_{2}$, and $\xi$, we can calculate the constants $D_{3}^{(i)}$ and $D_{4}^{(i)}$, $(i=1,2)$ from (30). After that, we can calculate the bending moments (26) and the normal forces (27).

\subsection{Kinematically Admissible Solution and Optimality Conditions}

In this part of the paper, the boundary conditions of the shell (37) and the condition of the compatibility of displacements rates (38) will be used (see Figure 5)

$$
\begin{gathered}
x=0 ; \dot{w}_{x}^{(1)}=0 ; \\
x=x_{1} ; \dot{w}_{x}^{(1)}=\dot{w}_{x}^{(2)} .
\end{gathered}
$$

The conditions (37) and (38) reads

$$
\begin{gathered}
D_{1}^{(1)} \sin \omega^{(1)} x+D_{2}^{(1)} \cos \omega^{(1)} x=0,(x=0), \\
D_{1}^{(1)} \sin \omega^{(1)} x_{1}+D_{2}^{(1)} \cos \omega^{(1)} x_{1}=D_{1}^{(2)} \sin \omega^{(2)} x_{1}+D_{2}^{(2)} \cos \omega^{(2)} x_{1} .
\end{gathered}
$$

Two equations with four unknown variables $D_{1}^{(1)}, D_{2}^{(1)}, D_{1}^{(2)}$ and $D_{2}^{(2)}$ are obtained, and using (31) and (32) it follows as

$$
D_{2}^{(1)}=0 \text {; }
$$




$$
D_{1}^{(1)} \sin \left(X_{1} \sqrt{\frac{2}{E_{1}}}\right)=D_{1}^{(2)} \sin \left(X_{1} \sqrt{\frac{2}{E_{2}}}\right)+D_{2}^{(2)} \cos \left(X_{1} \sqrt{\frac{2}{E_{2}}}\right) .
$$

Application of Optimum Conditions

The conditions of optimum (18), derived in Section 3. for this case, will take the form

$$
\begin{aligned}
& \frac{\partial \mathcal{D}_{1}}{\partial e_{1}}=A_{1} \frac{d c_{1}}{d e_{1}}, \\
& \frac{\partial \mathcal{D}_{2}}{\partial e_{2}}=A_{2} \frac{d c_{2}}{d e_{2}} .
\end{aligned}
$$

For a specific cost, we choose $c_{1}=e_{1}$ and $c_{2}=e_{2}$, which leads to the shell of minimal volume and minimal weight. From Equations (43) and (44) it follows

$$
\begin{gathered}
\frac{\partial \mathcal{D}_{1}}{\partial e_{1}}=2 \pi R x_{1} . \\
\frac{\partial \mathcal{D}_{2}}{\partial e_{2}}=2 \pi R\left(x_{2}-x_{1}\right) .
\end{gathered}
$$

By substituting $m_{x}$ and $n_{\theta}$ from (3) and (4) and $\dot{k}_{x}, \dot{\lambda}_{\theta}$ and $\dot{w}_{x}$ from (6) and (28) into the expression (5) of the power of dissipation energy of the ring of thickness $e_{1}, \mathcal{D}_{1}$ is obtained as

$$
\begin{aligned}
& \mathcal{D}_{1}=\left(\frac{\pi R \sigma_{0} e_{1}^{2} \omega^{(1)}}{2}+\frac{2 \pi \sigma_{0} e_{1}}{\omega^{(1)}}\right)\left[D_{1}^{(1)} \cos \left(\omega^{(1)} x_{1}\right)-D_{2}^{(1)} \sin \left(\omega^{(1)} x_{1}\right)\right]- \\
& \frac{\pi R \sigma_{0} e_{1}^{2}}{2}\left[D_{1}^{(2)} \omega^{(2)} \cos \left(\omega^{(2)} x_{1}\right)-D_{2}^{(2)} \omega^{(2)} \sin \left(\omega^{(2)} x_{1}\right)\right]-\frac{2 \pi \sigma_{0} e_{1}}{\omega^{(1)}} D_{1}^{(1)} .
\end{aligned}
$$

The optimum condition (45) with the dissipation $\mathcal{D}_{1}$ from (47) becomes

$$
\begin{gathered}
\bar{D}_{1}^{(1)}\left[4.5 \sqrt{\frac{E_{1}}{2}} \cos \left(X_{1} \sqrt{\frac{2}{E_{1}}}\right)+1.5 X_{1} \sin \left(X_{1} \sqrt{\frac{2}{E_{1}}}\right)-3 \sqrt{\frac{E_{1}}{2}}\right]+\bar{D}_{2}^{(1)}\left[1.5 X_{1} \cos \left(X_{1} \sqrt{\frac{2}{E_{1}}}\right)-4.5 \sqrt{\frac{E_{1}}{2}} \sin \left(X_{1} \sqrt{\frac{2}{E_{1}}}\right)\right]- \\
\bar{D}_{1}^{(2)} E_{1} \sqrt{\frac{2}{E_{2}}} \cos \left(X_{1} \sqrt{\frac{2}{E_{1}}}\right)+\bar{D}_{2}^{(2)} E_{1} \sqrt{\frac{2}{E_{2}}} \sin \left(X_{1} \sqrt{\frac{2}{E_{2}}}\right)-2 X_{1}=0 \\
\text { where the notation for constants } \bar{D}_{i}^{(i)} \text { was introduced } \\
\qquad \bar{D}_{i}^{(i)}=\frac{\sigma_{0}}{R} D_{i}^{(i)}, i=1,2 \\
\text { Due to the symmetry of the shell, only half of it was considered, as well as half of } \\
\text { the dissipation } \mathcal{D}_{2} \text { of the central ring. By analogous procedure as for } \mathcal{D}_{1} \text {, a dissipation } \mathcal{D}_{2} \\
\text { was obtained } \\
\mathcal{D}_{2}=D_{1}^{(2)}\left[\frac{2 \pi \sigma_{0} e_{2}}{\sqrt{\frac{2}{e_{2} R}}} \cos \left(\sqrt{\frac{2}{e_{2} R}} x_{2}\right)-\frac{2 \pi \sigma_{0} e_{2}}{\sqrt{\frac{2}{e_{2} R}}} \cos \left(\sqrt{\frac{2}{e_{2} R}} x_{1}\right)+\frac{\pi R \sigma_{0} e_{2}^{2}}{2} \sqrt{\frac{2}{e_{2} R}} \cos \left(\sqrt{\frac{2}{e_{2} R}} x_{2}\right)\right]+ \\
D_{2}^{(2)}\left[-\frac{2 \pi \sigma_{0} e_{2}}{\sqrt{\frac{2}{e_{2} R}}} \sin \left(\sqrt{\frac{2}{e_{2} R}} x_{2}\right)+\frac{2 \pi \sigma_{0} e_{2}}{\sqrt{\frac{2}{e_{2} R}}} \sin \left(\sqrt{\frac{2}{e_{2} R}} x_{1}\right)-\frac{\pi R \sigma_{0} e_{2}^{2}}{2} \sqrt{\frac{2}{e_{2} R}} \sin \left(\sqrt{\frac{2}{e_{2} R}} x_{2}\right)\right] .
\end{gathered}
$$

With (50) and the optimality condition (46), the fourth equation (51) was obtained

$$
\begin{aligned}
& \bar{D}_{1}^{(2)}\left[4.5 \sqrt{\frac{E_{2}}{2}} \cos \left(X_{2} \sqrt{\frac{2}{E_{2}}}\right)-3 \sqrt{\frac{E_{2}}{2}} \cos \left(X_{1} \sqrt{\frac{2}{E_{2}}}\right)+1.5 X_{2} \sin \left(X_{2} \sqrt{\frac{2}{E_{2}}}\right)-X_{1} \sin \left(X_{1} \sqrt{\frac{2}{E_{2}}}\right)\right)+ \\
& \bar{D}_{2}^{(2)}\left(3 \sqrt{\frac{E_{2}}{2}} \sin \left(X_{1} \sqrt{\frac{2}{E_{2}}}\right)-4.5 \sqrt{\frac{E_{2}}{2}} \sin \left(X_{2} \sqrt{\frac{2}{E_{2}}}\right)+1.5 X_{2} \cos \left(X_{2} \sqrt{\frac{2}{E_{2}}}\right)-X_{1} \cos \left(X_{1} \sqrt{\frac{2}{E_{2}}}\right)\right]-2\left(X_{2}-X_{1}\right)=0 .
\end{aligned}
$$


The system of the linear Equations (41), (42), (48) and (51) is simply to solve and calculate the unknown constants $\bar{D}_{1}^{(1)}, \bar{D}_{2}^{(1)}, \bar{D}_{1}^{(2)}$ and $\bar{D}_{2}^{(2)}$. Finally, the displacements rates $\dot{w}_{x}^{(1)}$ and $\dot{w}_{x}^{(2)}$ can be obtained by

$$
\dot{w}_{x}^{(i)}=D_{1}^{(i)} \sin \omega^{(i)} x+D_{2}^{(i)} \cos \omega^{(i)} x,(i=1,2),
$$

and the collapse mechanism is determined. In this way, a complete solution in the sense of the theory of limit analysis, including the statically and kinematically parts, can be determined.

\section{Numerical Examples}

A full cylindrical shell composed of rings of different thicknesses is simply supported and subjected to the uniform internal pressure $p_{0}$, as in Figure 2 . The following initial data as dimensionless values are given as ring length $X_{1}$, then $X_{2}$, and $P_{s}=p_{0} / \sigma_{0}$. The values of optimal ring thicknesses $E_{1}$ and $E_{2}$ were determined according to the procedure given in Section 4.

Example 1. In this example, the dimensioning of the shell is calculated with the given data $X_{1}=0.08, X_{2}=0.16$, and $P_{s}=0.035$. In the applied numerical procedure, the process is interrupted with the criterion of achieved accuracy $\varepsilon=0.0001$. Table 1 shows the last iteration in which $\xi$ was varied in the interval $(0.743 ; 0.742)$ with $n=10$.

Table 1. Last iteration for $\xi$ of the numerical procedure.

\begin{tabular}{llllll}
\hline $\boldsymbol{\zeta}$ & $\boldsymbol{E}_{\mathbf{2}}$ & $\boldsymbol{E}_{\mathbf{1}}$ & $\boldsymbol{\xi} \cdot \boldsymbol{E}_{\mathbf{2}} \cdot \boldsymbol{E}_{\mathbf{2}}$ & $\boldsymbol{E}_{\mathbf{1}} \cdot \boldsymbol{E}_{\mathbf{1}}$ & VOLUME \\
\hline 0.7429 & 0.03073 & 0.02635 & 0.000701410 & 0.000694219 & 0.004566007 \\
0.7428 & 0.03072 & 0.02636 & 0.000701118 & 0.000695064 & 0.004566942 \\
0.7427 & 0.03071 & 0.02638 & 0.000700826 & 0.000695907 & 0.004567874 \\
0.7426 & 0.03071 & 0.02640 & 0.000700534 & 0.000696753 & 0.004568809 \\
0.7425 & 0.03071 & 0.02641 & 0.000700242 & 0.000697590 & 0.004569744 \\
0.7424 & 0.03071 & 0.02643 & 0.000699947 & 0.000698453 & 0.004570687 \\
0.7423 & 0.03070 & 0.02644 & 0.000699653 & 0.000699302 & 0.004571624 \\
0.7422 & 0.03070 & 0.02646 & 0.000699363 & 0.000700150 & 0.004572559 \\
0.7421 & 0.03069 & 0.02648 & 0.000699072 & 0.000700999 & 0.004573497 \\
\hline
\end{tabular}

The step in that iteration was $h_{3}=10^{-4}$ and the numerical procedure was completed when $\varepsilon=0.0001$ was reached. For $\xi=0.7422$, the ring thicknesses $E_{1}=0.02646$ and $E_{2}=0.0307$ were calculated. The minimum volume is $\bar{V}=0.009145$ where it is $\bar{V}=\frac{V}{2 \pi R^{3}}$. The displacement rates $\dot{w}_{i}(i=1,2)$ and its slopes $P_{i}(i=1,2)$ corresponding to the circular plastic hinges of the shell collapse mechanism were calculated (see Table 2).

In this way, the kinematic part of the solution of this example is satisfied.

Bearing in mind that dimensionless quantities were taken into account in the calculation, we assume that it is $\sigma_{0}=235 \mathrm{Mpa}$ and the radius of the shell $\mathrm{R}=2 \mathrm{~m}$. The thickness of the ring near the support is $e_{1}=52.92 \mathrm{~mm}$, the thickness of the central ring is $e_{2}=61.4 \mathrm{~mm}$, and imposed limit load $p_{0}=8.225 \mathrm{Mpa}$.

Example 2. The following examples show the results of the optimal design of two shells with rings of different lengths, subjected by the same limit load $p_{0}=0.0345 \sigma_{0}$. The data for the first shell is $X_{1}=0.085$ and $X_{2}=0.17$ and for the second $X_{1}=0.09$ and $X_{2}=0.18$. In the upper part of Table 3 , we represent the calculated thicknesses of these shells $E_{1}$ and $E_{2}$ and case when $X_{1}=0$ for the shells of constant thicknesses $E=E_{2}$. 
Table 2. The strain rate velocities and their slopes.

\begin{tabular}{cccc}
\hline $\boldsymbol{X}_{\mathbf{1}}$ & $\dot{\boldsymbol{w}}_{1}$ & $\boldsymbol{X}_{\mathbf{2}}$ & $\dot{\boldsymbol{w}}_{2}$ \\
\hline 0.00000000 & 0.00000000 & 0.08000000 & -17.97580596 \\
0.00800000 & -1.94955594 & 0.08800000 & -19.08173715 \\
0.01600000 & -3.88968472 & 0.09600000 & -20.108173715 \\
0.02400000 & -5.81100477 & 0.10400000 & -21.05072739 \\
0.03200000 & -7.70422548 & 0.11200000 & -21.90557981 \\
0.04000000 & -9.56019208 & 0.12000000 & -22.66913126 \\
0.04800000 & -11.36992999 & 0.12800000 & -23.33819928 \\
0.05600000 & -13.12468814 & 0.13600000 & -23.90999527 \\
0.06400000 & -14.81598132 & 0.14400000 & -24.38213601 \\
0.07200000 & -16.43563122 & 0.15200000 & -24.75265364 \\
0.08000000 & -17.97580596 & 0.16000000 & -25.02000388 \\
\hline $\boldsymbol{X}_{\mathbf{1}}$ & $\boldsymbol{P}_{\mathbf{1}}$ & $\boldsymbol{X}_{\mathbf{2}}$ & $\boldsymbol{P}_{\mathbf{2}}$ \\
\hline 0.000 & -244 & 0.08 & -136 \\
0.08 & -187 & 0.16 & -22 \\
\hline
\end{tabular}

Table 3. The calculated thicknesses of optimal shells.

\begin{tabular}{ccccc}
\hline \multicolumn{5}{c}{$\boldsymbol{P}_{\boldsymbol{s}}=\mathbf{0 . 0 3 4 5}$} \\
\hline$X_{1}$ & 0 & 0.09 & 0 & 0.085 \\
$X_{2}$ & 0.18 & 0.18 & 0.17 & 0.17 \\
$E_{1}$ & - & 0.02861 & - & 0.02755 \\
$E_{2}$ & 0.0321 & 0.033 & 0.031 & 0.0321 \\
\hline \multicolumn{5}{c}{$\boldsymbol{P}_{\boldsymbol{s}}=\mathbf{0 . 0 3 5}$} \\
\hline$X_{1}$ & 0 & 0.07 & 0.095 & 0.11 \\
$X_{2}$ & 0.19 & 0.19 & 0.19 & 0.19 \\
$E_{1}$ & - & 0.02638 & 0.02961 & 0.03096 \\
$E_{2}$ & 0.0332 & 0.034 & 0.0345 & 0.0344 \\
\hline
\end{tabular}

Example 3. The examples of the shells with the same limit load $P_{S}=0.035 \sigma_{0}$ and the same length $\bar{L}=2 X_{2}$, but different ring lengths, were calculated (See Table 3, lower part). The following data for $X_{1}$ : 0 (the shell of constant thickness); 0.7; 0.095 and 0.11 have been taken. Comparing these shells, we conclude that the shell with a constant thickness has a maximum volume, $\bar{V}=0.012616$.

\section{Results and Discussion}

Performing the structural optimization, the objective function is chosen to be connected with the variables of dimensioning, i.e., the ring thicknesses $e_{1}$ and $e_{2}$. It was necessary for optimization to be connected with the determination of the minimum of energy and total power of dissipation energy of rings of the shell that the conditions of optimum, derived in Section 3, provide. Through the limit analysis, the greatest resistance of the shell is used. While the shell is operating at the limits of its resistance, we perform a variation of the variable dimensioning, during the derivation of the condition of optimum, to reach the minimum of the energy of dissipation. Thus, the "best" solution that works with the least material under the limit load $p_{0}$ was determined. In the condition of optimum, the total power of dissipation energy depends only on bending moments $M_{x}, M_{p x}$, normal forces $N_{\theta}, N_{p}$, and on-field of displacement rates $\dot{w}_{x}^{(i)}$ which characterize the collapse mechanism.

In the optimal plastic design (Section 4), both analytical and numerical approaches are exposed. To solve this nonlinear problem (Equations (33)-(35)), we start from the fact that it is real and practical. For approximate initial values, the thickness $e$ from the shell of constant thickness was used, as well as the condition $e_{1} \leq e_{2}$. Thus, the initial value of $E_{2}$ of the central ring thickness was chosen in the subroutine as very small in relation to the shell of constant thickness $e$. As the number of steps increases, the interval in which the unknown $E_{2}$ is located occurs. The determination of the thickness $E_{1}$ was carried out 
in a similar way. As explained in Section 4.1, the main program decreases the interval of parameter $\xi$ at the point where the difference $\xi \cdot E_{2}^{2}-E_{1}^{2}$ from (35) change sign and specifies the new interval of $\xi$. In Example 1, (Table 1), this change in sign would be between $\xi=0.7423$ and $\xi=0.7422$. In this particular case, the interval is reduced because accuracy is required; $\varepsilon=0.0001$ is reached and this procedure is interrupted.

Based on the same Example 1 and Table 2, a diagram of the displacement rates $\dot{w}_{x}^{(i)}$ as well as the slopes, i.e., tangents, on the plastic hinges are shown (see Figure 6). Thus, the collapse mechanism of this shell was verified.

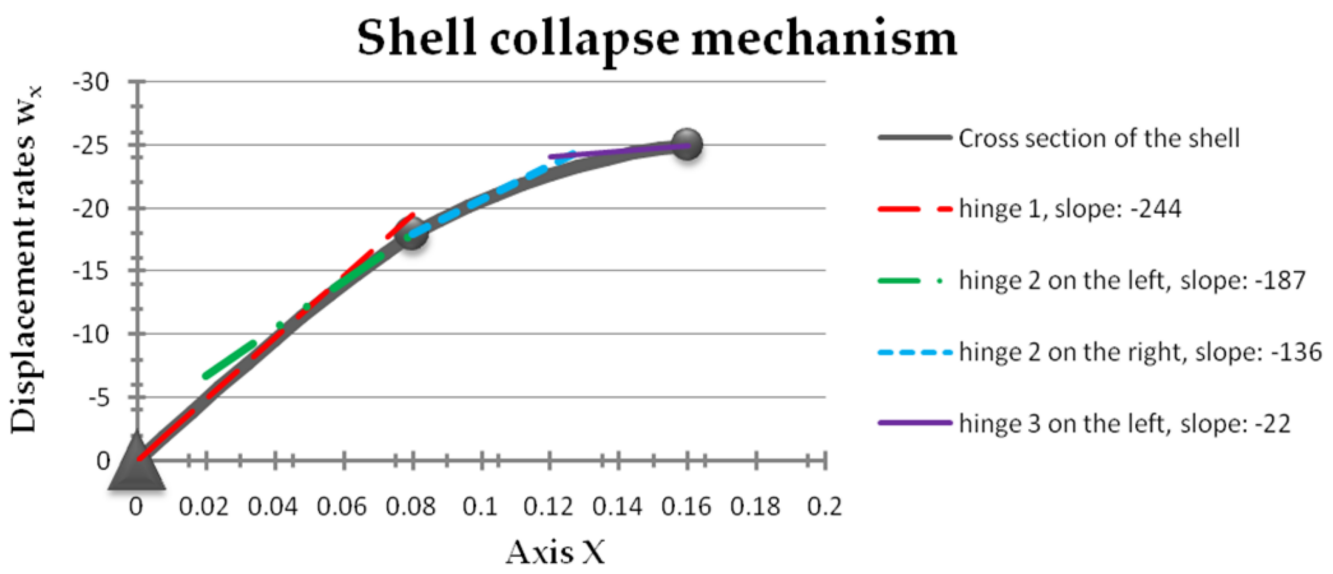

Figure 6. The simply supported full shell composed of three rings.

In Example 3, three shells with rings were considered. Although each of these shells with rings for a set value of length $X_{1}$ is optimal in the plastic range, its gains of volumes (and weights) in relation to the shell of constant thickness are different as follows: for $X_{1}=0.07, g_{1}=6.43539 \%$; for $X_{1}=0.095, g_{2}=3.571987 \%$; for $X_{1}=0.11, g_{3}=2.4425 \%$.

The minimal volume, (i.e., the weight) was obtained for a shell with ring length $X_{1}=0.07$. We conclude that by choosing the length $X_{1}$ we can obtain a generally optimal volume, i.e., the optimal weight for the imposed limit load.

\section{Conclusions}

- The necessary and sufficient conditions of the optimum for the full circular cylindrical shell with technological limitations, composed of rings of variable thicknesses have been derived with one behavioral constraint related to a specific constitutive low, for limit load-carrying capacity $p_{0}$. The paper proves that the necessary optimality conditions are identical to the sufficient conditions of the optimum. The objective function is $\operatorname{cost} C$. The specific $\operatorname{cost} c_{i}$ is the function of thickness $e_{i}$ which leads to the shell of minimal volume, and also minimal weight. These conditions are derived for a more complex case, i.e., for a full shell with technological constraints, not a sandwichtype shell. Note that in [23], Schielld was the first to derive optimal conditions in the plastic region for a sandwich-type shell, whose thickness can vary continuously from zero to infinity.

- The optimal structural design of shell (in detail with three rings) with an analytical approach complemented by numerical tools and direct connection with basic equations of limit analysis (Section 2) is given. In the static part, the problem is reduced to three linear Equations (33)-(35) and the procedure for their numerical solution is briefly explained, which leads to the optimal dimensions of the shell. Within the kinematic part, the energy dissipation of rings $\mathcal{D}_{1}$ and $\mathcal{D}_{2}$ were analytically obtained, and, using the optimum conditions, the displacement rates of the shell $\dot{w}_{x}$ were determined. In this way, the real collapse mechanism is defined with a minimum of dissipative shell energy. This numerical procedure was applied, and the dimensions in numerical examples were obtained. 
- In numerical examples, the optimal dimensions of shells were successfully calculated, applying the conditions of optimum derived in Section 3. Due to the choice of the cost function $C, c_{i}=e_{i}$, the shells have minimum volume. In Example 1, the collapse mechanism is shown and confirmed (see Figure 6). For shells of the same limit load $p_{0}$ and the same length $L$, the length of ring $X_{1}$ is varied. By comparing these shells, for technological reasons, composed of three rings, with an optimal shell of constant thickness, a gain of $6.4 \%$ was achieved. This gain was for the shell of length $X_{1}=0.07$. By further calculation, the most favorable length $X_{1}$ can be obtained.

- This type of cylindrical shell constituted of rings is common in the mechanical, chemical industry, etc. With the increase in the number of rings, the gains also increase. Due to complexity, there are very few solutions to this problem given in analytical, closed-form, and only for simple cases with the results of strongly simplifying assumptions are obtained. The solutions in this paper, which are mostly analytical and complemented by exposed numerical tools, can be useful for confirming numerical procedures, as well as providing information for creating concepts and ideas for technological solutions to more practical and complex problems.

- In further work, this problem will be extended, especially, to the cases of shells with stiffeners, then with end loads for more complex problems of stability and dynamics from practice. Based on the results presented in this paper, the shells, differing in terms of the limit conditions, types of loads, and the number of rings of various thicknesses may be the object of new research and experimental verification to obtain new results.

Author Contributions: Conceptualization, D.T. and T.I.; methodology, D.T.; software, T.I. and A.I.; validation, D.T., T.I. and S.Ž.; formal analysis, M.S.Š.; investigation, A.I.; resources, T.I.; data curation, T.I.; writing-original draft preparation, D.T. and T.I.; writing-review and editing, D.T.; visualization, M.S.Š.; supervision, S.Ž. and M.S.Š. All authors have read and agreed to the published version of the manuscript.

Funding: This research received no external funding.

Conflicts of Interest: The authors declare no conflict of interest.

\section{References}

1. Farshad, M. Design and Analysis of Shell Structures; Springer: Berlin/Heidelberg, Germany, 2010.

2. Farkas, J.; Jarmal, K. Optimum Design of Steel Structures; Springer: Berlin/Heidelberg, Germany, 2013.

3. Kouam, M. Contribution a L'analyse Limite eau Dimensionnement Optimal des Coques Cylindriques. Ph.D. Thesis, Faculte Polytechnique, Palaiseau, French, 1983.

4. Caseiro, J.F.; Andrade-Campos, A. An Evolutionary-Inspired Optimization Algorithm Suitable for Solid Mechanics Engineering Inverse Problems. Int. J. Mechatron. Manuf. Syst. 2011, 4, 415-440. [CrossRef]

5. Rocha, T.M.; Meireles, J.F.; Pinho, A.C.; Ambrósio, J.A. Update of Coarse Finite Elements Structural Models for Dynamic Analysis Identified from Reference Responses. Int. J. Mechatron. Manuf. Syst. 2011, 4, 402-414. [CrossRef]

6. Save, M.; Igić, T. Examples of double-purpose optimal beams. J. Theor. Appl. Mech. 1982, 1, 311-321.

7. Save, M.; Prager, W. Structural Optimization, Vol. 1. Optimality Criteria; Plenum Press: New York, NY, USA, 1985.

8. Karpat, F. A Virtual Tool for Minimum Cost Design of a Wind Turbine Tower with Ring Stiffeners. Energies 2013, 6, 3822-3840. [CrossRef]

9. Radu, D.; Sedmak, A.; Sedmak, S.; Dunjić, M. Stress Analysis of Steel Structure Comprising Cylindrical Shell with Billboard Tower. Teh. Vjesn. 2018, 25, 429-436. [CrossRef]

10. Cui, J.; Zheng, Y.; Ohsaki, M.; Luo, Y. Shape Optimization of Piecewise Free-form grid Surface using Plate Components. Eng. Struct. 2021, 245, 112865. [CrossRef]

11. Krejsa, M.; Seitl, S.; Brozovsky, J.; Lehner, P. Fatigue damage prediction of short edge under various load: Direct Optimized Probabilistic Calculation. In Proceedings of the 2nd International Conference on Structural Integrity, ICSI 2017, Funchal, Portugal, 4-7 September 2017.

12. Wang, D.; Qin, X.; Chen, W.; Wang, S. Simulation of the Deformation and Failure Characteristics of a Cylinder Shell under Internal Explosion. Appl. Sci. 2022, 12, 1217. [CrossRef]

13. Hamouche, W. Contrôle de Forme de Coques Multi stables: Modélisation, Optimisation et Miseen Euvre. Ph.D. Thesis, Université Pierre et Marie Curie Paris VI, Paris, France, 2016.

14. Fraldi, M.; Guarracino, F. An Improved Formulation for the Assessment of the Capacity Load of Circular Rings and Cylindrical Shells under External Pressure. Part1. Analytical derivation. Thin-Walled Struct. 2011, 49, 1054-1061. [CrossRef] 
15. Ambati, M.; Kiendl, J.; DeLorensis, L. Isogeometric Kirchhoff-Love Shell Formulation for Elasto-Plasticity. Comput. Methods Appl. Mech. Eng. 2018, 340, 320-339. [CrossRef]

16. Tokarev, A.S.; Karetnikov, D.V.; Fayrushin, A.M. Determining Optimal Geometric Dimensions of Alternative Design Elements of Rolled and Welded Tube-to-Tube Sheet Joints. In Proceedings of the International Conference on Industrial Engineering (ICIE2020), Sochi, Russia, 18-21 May 2020; pp. 227-233.

17. Filatov, V.H. Optimal Design of Single-Layered Reinforced Cylindrical Shells. J. Mech. Eng. 2021, 24, 58-64. [CrossRef]

18. Zheng, Y.; Zhang, J.; Ohsaki, M.; Wan, H.; Yang, C.; Luo, Y. Optimization of the rod forces in the reticular support structure of JUNO central detector. Structures 2021, 33, 1645-1658. [CrossRef]

19. Bideq, M.; Boussitine, L.; Guerlement, G. Contribution à l'optimisation des coques sphériques par l'analyse limite et les algorithmes génétiques. In Proceedings of the 23ènie Congrès de Méchanique, Lille, France, 28 September 2017; Available online: https:/ / hal.archives-ouvertes.fr/hal-03465803/document (accessed on 1 January 2022).

20. Drucker, D.C. Limit Analysis of the Cylindrical Shells under Axially-symmetric Loading. In Proceedings of the 1st Midwestern Conference Solid Mechanics, Urbana, IL, USA, 24-25 April 1953.

21. Hodge, P. Plastic Analysis of Structures; McGraw-Hill: New York, NY, USA, 1959.

22. Igić, T.S. Contribution to the Optimum Design of Structures. Ph.D. Thesis, Civil Engineering Faculty, University of Nis, Nis, Serbia, 1980.

23. Shield, R.T. On the optimum design of shells. J. Appl. Mech. 1960, 27, 316-322. [CrossRef] 